\title{
Fibromatosis - immunohistochemical evaluation, differential diagnosis from gastrointestinal tumors, and other mesenchymal tumours
}

\author{
Dorota E. Lacka1, Anna Nasierowska-Guttmejer ${ }^{2}$ \\ ${ }^{1}$ Department of Pathology, Central Clinical Hospital of the MSWiA, Warsaw, Poland \\ 2Department of Pathology, Jan Kochanowski University, Kielce, Poland
}

Gastroenterology Rev 2019; 14 (1): 79-85

DOI: https://doi.org/10.5114/pg.2019.83429

Key words: fibromatosis, gastrointestinal stromal tumor, immunostainings, $\beta$-catenin, desmoid.

Address for correspondence: Dorota E. Lacka, Department of Pathology, Central Clinical Hospital of the MSWiA, 137 Wołoska St, 02-507 Warsaw, Poland, phone: +48 506324 903, e-mail: dorotalacka@gmail.com

\begin{abstract}
Introduction: Fibromatosis is a histologically benign growth of fibroblastic and myofibroblastic cells, with a potential to recur and invade local organs. It can occur as a superficial or deep form. Visceral fibromatosis and superficial fibromatosis are histologically similar. They both have alterations in the WNT signalling pathway, but mutations in the APC or $\beta$-catenin gene do not occur in superficial fibromatoses.

Aim: To present four cases of deep fibromatosis and one case of Peyronie's disease, along with immunohistochemical staining analysis and the criteria for differential diagnosis.

Material and methods: All patients were hospitalised in the Central Clinical Hospital of the MSWiA in Warsaw during the period of 2012-2015. Surgical specimens were examined, and tissue samples were embedded in paraffin blocks.

Results: As the result of the study we present a short algorithm of immunostainings that can be useful in differential diagnosis. When a spindle cell tumour is encountered in the abdomen a gastrointestinal stromal tumor (GIST) should always be excluded; therefore, a CD117 staining is recommended as the first step. When the staining is negative, fibromatosis can be taken into consideration. $\beta$-Catenin staining should be done in order to confirm that diagnosis.

Conclusions: The diagnosis of fibromatosis is not always simple; GISTs can easily be mistaken for it. Immunohistochemical staining with CD34 and CD117 antibodies are useful in differential diagnosis. DTF should present negative stainings for S100, CD34, CD99, and bcl-2, which can help to distinguish it from other mesenchymal tumours.
\end{abstract}

\section{Introduction}

Fibromatosis is a histologically benign growth of fibroblastic and myofibroblastic cells, with a potential to recur and invade local organs. It can occur as a superficial or deep (desmoid) form. The term desmoid, introduced in 1838 by Muller, comes from the Greek word "desmos" (tendon-like) and describes the nature of this disease [1,2].

Deep, visceral fibromatosis and superficial fibromatosis are histologically similar. They both have alterations in the WNT signalling pathway, but mutations in the adenomatous polyposis coli (APC) or $\beta$-catenin gene do not occur in superficial fibromatoses [3]. The clinical course is also different because superficial fibromatoses usually remain small and do not have a tendency to recur whereas deep fibromatoses (DF) often display a locally invasive character [4].
The aetiopathogenesis of fibromatosis still remains unclear. The precursor cell is probably a progenitor mesenchymal cell, while trauma, oestrogen hormone, and pregnancy play a role in the aetiopathogenesis of this tumour [5]. The molecular mechanisms responsible for this disease remain unknown, but results of recent studies suggest that $\beta$-catenin may be a significant factor.

Deep (also called aggressive) fibromatosis is classified into three forms: abdominal, when it occurs in anterior abdominal wall or rectum, happens mainly in relation to pregnancy; extra the abdominal form can involve the thorax, the shoulder, the thigh, or the head and neck region; and the third type, intraabdominal fibromatosis, which may occur in the mesentery or intrapelvic space [1, 2].

Although desmoid-type fibromatosis in most cases occurs sporadically, $15 \%$ of cases are associated with FAP Gardner syndrome. Gardner fibromas are considered as 
precursor lesions. Desmoids related to Gardner syndrome most commonly develop intra-abdominally [3].

Catenin (cadherin-associated protein) (CTNNB1), also called $\beta$-catenin, is a dual-function protein, regulating the coordination of cell-cell adhesion and gene transcription. In humans, the CTNNB1 protein is encoded by the CTNNB1 gene. $\beta$-Catenin is a subunit of the cadherin protein complex and acts as an intracellular signal transducer in the Wnt signalling pathway $[4,6]$.

Carcinogenesis in the Wnt pathway can result in mutations of the $\beta$-catenin gene and nuclear accumulation of the protein. These mutations are associated with many cancers, including hepatocellular carcinoma, colorectal carcinoma, lung cancer, malignant breast tumours, and ovarian and endometrial cancer. $\beta$-Catenin is regulated and destroyed by the $\beta$-catenin destruction complex, and in particular by the APC protein, encoded by the tumour-suppressing APC gene. Genetic mutation of the APC gene is also strongly linked to colorectal cancer resulting from familial adenomatous polyposis.

Usually, in patients with DF, the presence of $\beta$-catenin mutation excludes simultaneous presence of the APC mutation and vice versa.

Even though superficial fibromatoses lack the mutation in the $\beta$-catenin gene, nuclear accumulation of $\beta$-catenin has been reported in up to $86 \%$ of superficial fibromatoses involving, in most cases, a minority of nuclei (mean: 13\%). Desmoid tumours show 60-100\% of nuclear staining in almost every case [4]. The significance of focal nuclear accumulation of $\beta$-catenin in superficial fibromatosis remains unclear. It has been reported that the presence of specific (S45F) CTNNB1 mutation is predictive for recurrence in patients after surgical treatment for sporadic extra-abdominal and abdominal desmoids $[4,6,7]$. In the case of deep fibromatosis $\beta$-catenin staining is necessary, to exclude simple fibrosis and other conditions.

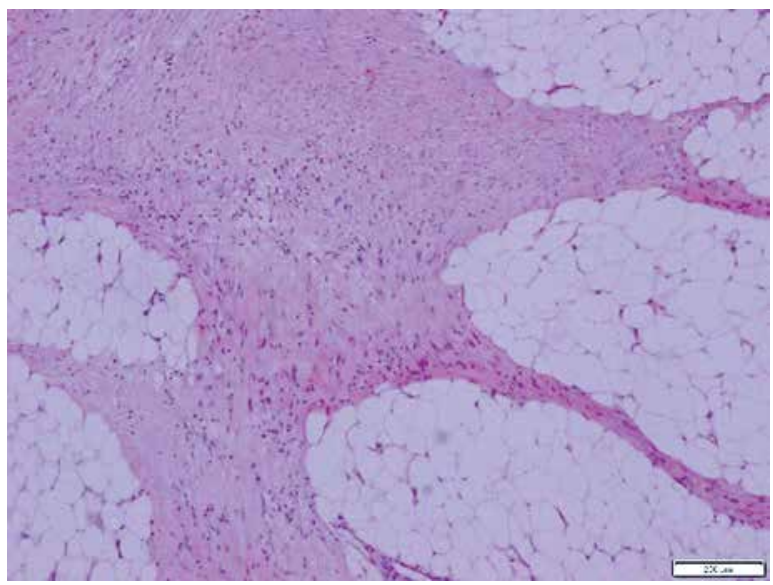

Figure 1. Case 1. Fibromatosis. Spindle cell neoplasm with ill-defined borders
Additionally, there have been reports of sex steroid receptor and COX-2 expression in desmoid-type fibromatosis [5]. Responses to single-agent therapy with anti-oestrogens and non-steroidal anti-inflammatory drugs are still unpredictable, and further research is still needed [7].

\section{Aim}

The aim of the study is to present differential diagnosis with immunohistochemical evaluation in cases of deep fibromatosis and a case of Peyronie's disease (plantar fibromatosis).

\section{Material and methods}

All patients were hospitalised in the Central Clinical Hospital of the Ministry of Internal Affairs (MSWiA) in Warsaw during the period 2012-2015. Surgical specimens were examined, and tissue samples were embedded in paraffin blocks. Three-micrometre-thick slides were prepared, stained with haematoxylin and eosin. For immunohistochemical evaluation DAKO stainings were used: Polyclonal Rabbit Anti-Human antibody CD 117 (diluted 1 : 500) A4502, Monoclonal Mouse antibody CD34 Class II IR 632 Clone aBEnd10, mouse monoclonal antibody caldesmon clone h-CD IR 054, mouse anti-human $\beta$-catenin IR 702 clone 1, monoclonal mouse anti-human SMA- $\alpha$ smooth muscle myosin heavy chain clone SMM S-1 IR 066, monoclonal mouse anti-human vimentin IR 630 clone V9, monoclonal mouse antihuman desmin IR 606 clone D33, oestrogen receptor alfa monoclonal mouse antihuman is 657 clone 1D5, mouse monoclonal antihuman progesterone receptor, polyclonal rabbit anti-s100 IR 504.

Case one referred to a 29-year-old woman who underwent surgery due to acute abdominal pain and a newly discovered tumour in her small bowel mesentery.

A 70-mm tumour, whitish and swirly on cut surface, was surgically removed.

On microscopic examination a mesenchymal tumour with fusiform cells without cytological atypia and without necrosis was described (Figure 1). Based on the location and type of growth, a primary diagnosis of gastrointestinal stromal tumors (GIST) was made. Immunohistochemical evaluation with CD117, CD34, and caldesmon was performed. All staining results were negative, and two mitotic figures in $50 \mathrm{HPF}$ were counted. The case was directed to the Oncology Centre in order to assess the c-kit mutation. The mutation was absent, and the tumour was misdiagnosed as a "wild type GIST".

A second diagnosis was performed in Baylor Medicine Hospital. The pathological report described a spin- 


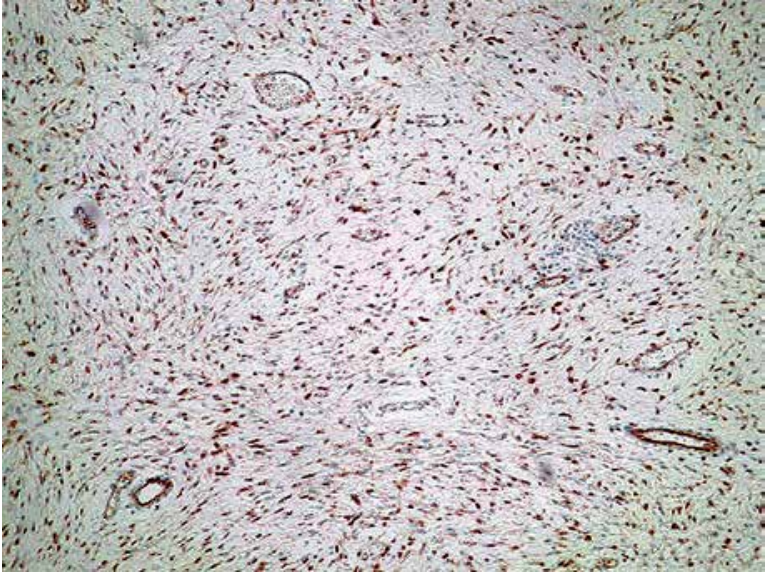

Figure 2. Case 1. $\beta$-Catenin-positive staining in most of the nuclei $(10 \times ; H+E)$

dle cell neoplasm with numerous blood vessels and poorly defined borders, extending to surgical margins. A final diagnosis of desmoid fibromatosis was made. Confirmatory immunostains such as $\beta$-catenin, SMA- $\alpha$, vimentin, and desmin, which are known to be positive in this tumour, were advised along with ruling out Gardner syndrome.

A panel of advised immunohistochemical markers was performed. The results were as follows: $\beta$-catenin staining showed a positive nuclear reaction (Figure 2 ), CD 117 staining was positive in single scattered cells (Figure 3), CD 34 staining was negative, SMA staining was positive, and desmin and S100 stainings were negative.

The oestrogen receptors were negative, and PR were focally positive.

The final diagnosis in CCH of MSWiA in Warsaw confirmed the diagnosis of fibromatosis. The patient was advised to perform evaluation for the FAP syndrome [8].

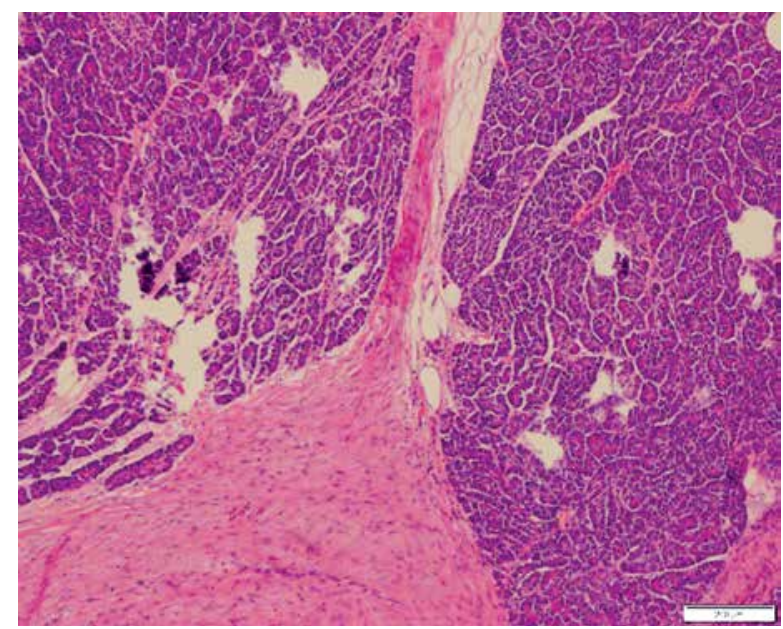

Figure 4. Case 2. Infiltration of fibromatosis tissue into the pancreas $(4 \times ; H+E)$

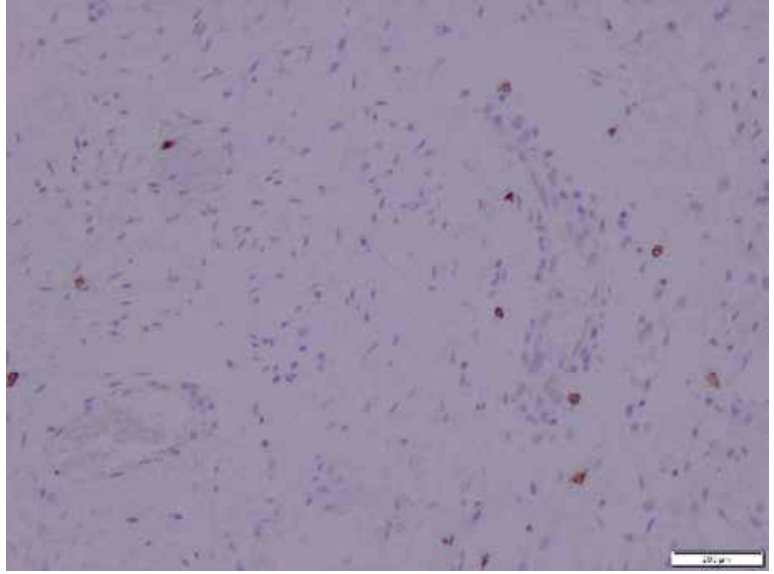

Figure 3. Case 1. Negative CD 117 staining. Positive reaction in single cells only

This case highlights the importance of immunohistochemical analysis in differential diagnosis of fibromatosis, and GIST and emphasises the role of the pathologist in interpretation of the results of molecular analysis and making an integrated diagnosis. It also shows that fibromatosis can clinically mimic a GIST tumour, and when diagnosing a GIST, fibromatosis should be excluded in differential diagnosis [9].

The second patient was a 43-year-old woman who was admitted to the hospital in order to surgically treat a pancreatic tail cyst. She had been suffering from abdominal pain for nine months and had been treated unsuccessfully for gastritis. Abdominal ultrasonography (USG) along with a computed tomography (CT) scan revealed a cystic structure in the pancreatic tail measuring about $73 \mathrm{~mm}$ and shaping the splenic artery.

The patient underwent surgery during which a cystic tumour of the pancreatic tail, which was infiltrating

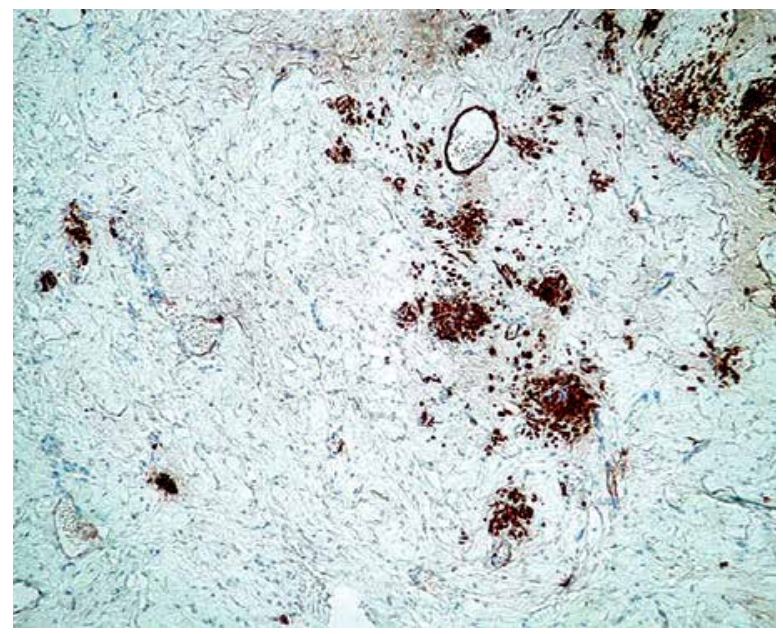

Figure 5. Case 2. Focal caldesmon positive staining $(4 x)$ 
the splenic flexure of the colon, the spleen, and the left renal capsule along with renal vessels, was detected. Distal resection of the pancreas with splenectomy and resection of the splenic colon flexure were performed along with left nephrectomy.

The Pathology Department received a spleen with an adjacent fragment of a pancreas and an adjacent fragment of a colon for further examination. A cyst, measuring $65 \mathrm{~mm}$ in diameter, was present in the region of the tail of the pancreas. In the space between the pancreas and the colon a fibrous area was detected. The kidney was sent separately, and on gross examination it was without any pathological findings.

On microscopic examination a diagnosis of mesenteric fibromatosis with a concomitant retention pancreatic cyst was made. The fibromatic tissue formed a 125 $\mathrm{mm}$ tumour localised in the distal part of the pancreas infiltrating the surrounding fat tissue of the pancreas (Figure 4) and the fat tissue surrounding the colon. The infiltration had also spread to the left adrenal gland. The tumour seemed to have been removed completely.

The differential diagnosis included other probable mesenchymal tumours such as gastrointestinal stromal tumour, a low-grade leiomyosarcoma and neurofibrosarcoma, as well as overabundant fibrosis.

Immunohistochemical stainings were performed, and the results were as follows: CD117 staining showed a positive reaction in single scattered cells, SMA, caldesmon (Figure 5), and desmin were focally positive, S100 was negative, and $\beta$-catenin showed positive nuclear staining.

Additionally, an evaluation of oestrogen and progesterone receptors was performed, and a positive reaction was observed in single cells for the OR $\alpha$, and for the PGR a focally positive reaction was observed.

This case was a rarely seen example of fibromatosis concomitant to a retention pancreatic cyst.

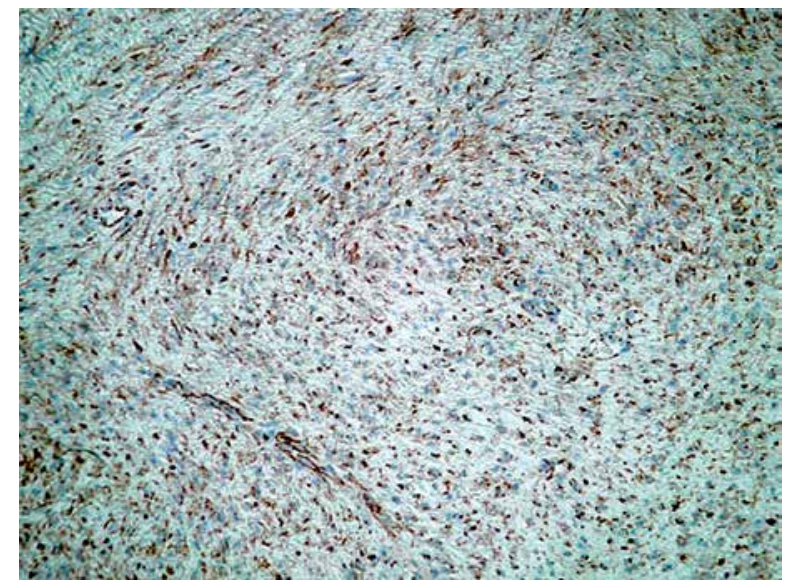

Figure 6. Case 5. Positive nuclear staining with $\beta$-catenin antibody (10x)
After the diagnosis of fibromatosis, the patient had been directed to a genetic clinic in order to rule out Gardner syndrome [8]. She had no family history of FAP and the results were negative for the APC germinal mutation.

The third case refers to a 40-year-old woman who was admitted to the hospital suffering from abdominal pain. She underwent surgery with a suspicion of a perforating small bowel tumour.

The pathology department received $450 \mathrm{~mm}$ of a small intestine with a $130 \mathrm{~mm}$ tumour in the bowel wall. The tumour embraced about 2/3 of the bowel's circumference, and it had a $20-\mathrm{mm}$ perforation that led to the lumen.

Microscopic examination detected a spindle cell tumour composed of bland cells without cytological atypia. It infiltrated the small bowel wall from the outside causing necrosis of the mucosa. A diagnosis of mesenteric desmoid fibromatosis was made. The tumour was present in the mesentery surgical margin. Margins of the bowel showed no signs of fibromatosis.

The diagnosis was confirmed immunohistochemically: CD117 staining showed a positive reaction in scattered single cells. Desmin, caldesmon, and SMA antibodies were negative, whereas vimentin was positive and $\beta$-catenin confirmed the diagnosis with positive nuclear staining. Evaluation of oestrogen and progesterone receptors was made, and the results were negative in both of the stainings.

This case highlights that although fibromatosis is a histologically benign tumour it can cause severe clinical complications such as bleeding or perforation.

The fourth patient was a 21-year-old man who was admitted to the hospital as an emergency case of gastrointestinal obstruction. He had a clinical history of appendectomy and several subsequent surgeries due to gastrointestinal obstruction.

The surgeons resected a fragment of the small bowel containing several intestinal loops in concretion. Gross examination revealed a thickened, fibrous wall of the bowel with inflammatory pseudopolyps of the mucosa and narrowing of the lumen.

On microscopic examination the fibromatous tissue was penetrating from the mesentery into the muscular wall of the small bowel. The tissue was also present in the resection margins.

A differential diagnosis between GIST and other mesenchymal tumours was performed. The GIST was excluded with a negative CD 117 staining.

The diagnosis of desmoid fibromatosis was confirmed immunohistochemically. $\beta$-Catenin nuclear staining was positive (Figure 6). Desmin and vimentin (Figure 7) stainings were positive, and S100 was negative. The oestrogen and progesterone receptors were only focally positive in single cells. This case is an excellent 
example that fibromatosis can be a recurring disease that is difficult to treat.

The fifth case was a 59-year-old woman who had a subcutaneous tumour resected from her sole. Multiple tissue fragments, measuring about $15 \mathrm{~mm}$, were sent for evaluation. The microscopic diagnosis of a superficial plantar fibromatosis was made.

$\beta$-Catenin staining to confirm the diagnosis was made. Cytoplasmic reaction was diffuse, and nuclear reaction was observed in about $50 \%$ of the cells (Figure 8 ).

This case presents a clinically benevolent form of a superficial fibromatosis. It also proves that even though superficial fibromatosis does not have the mutation in the $\beta$-catenin gene, in most of the cases at least focal positive nuclear $\beta$-catenin staining is observed (Table I).

\section{Discussion}

Gastrointestinal stromal tumors comprise most mesenchymal neoplasms of the gastrointestinal tract. Therefore, the first stage of diagnosis of a spindle cell tumour in the abdomen should confirm or exclude GIST. Unfortunately, overdiagnosis of GIST may occur. It is also advisable to remember that diagnosis of "wild type" GIST is a diagnosis of exclusion and can only be made after excluding other mesenchymal tumours, fibromatosis being one of them.

Fibromatosis is a rare disease that can manifest in a superficial form, which can be easily cured and rarely recurs, or in a deep aggressive form that can cause clinical complications (such as pain, bleeding, perforation or bowel obstruction) and is often recurring.

Visceral fibromatosis may take up various forms. It can manifest as a tumour, an ill-defined mass, or a cyst or it may not show in any form and only manifest because of complications such as bleeding or obstruction [1, 2].

The diagnosis is not always simple. Gastrointestinal stromal tumors can easily be mistaken for fibromatosis,

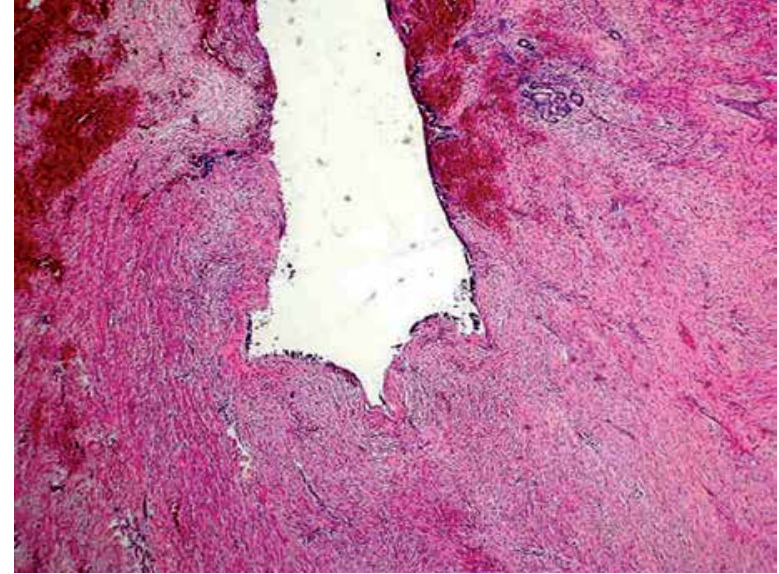

Figure 7. Case 3. Retention cyst in the pancreas. The wall of the cyst is composed of fibromatic tissue $(4 \times ; \mathrm{H}+\mathrm{E})$

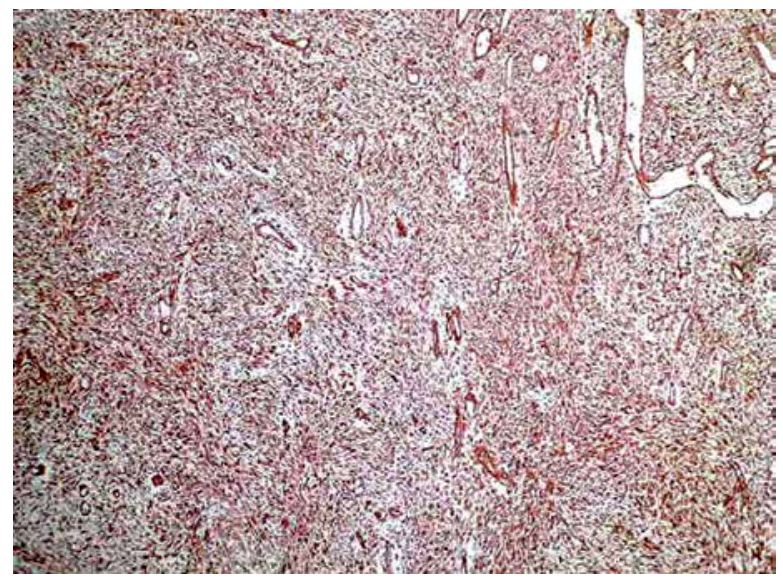

Figure 8. Case 4. Positive reaction with vimentin antibody $(4 \times)$

especially when the latter has focal growth. Deep visceral fibromatosis in young patients can raise suspicion of a wild type GIST and thus should be differentiated from it. Both GIST and fibromatosis can arise in the

Table I. Summary of staining results

\begin{tabular}{|c|c|c|c|c|c|c|c|c|c|}
\hline $\begin{array}{l}\text { Case } \\
\text { no. }\end{array}$ & $\begin{array}{l}\text { Location } \\
\text { of tumour }\end{array}$ & $\beta$-Catenin & CD117 & CD34 & $\begin{array}{l}\text { SMA/desmin } \\
\text { caldesmon }\end{array}$ & Vimentin & OR $\alpha$ & PGR & S100 \\
\hline 1 & $\begin{array}{l}\text { Mesentery } \\
\text { tumour }\end{array}$ & $(+)$ & $(-)$ & $(-)$ & SMA $(+), \operatorname{desmin}(-)$ & & - & Focal & - \\
\hline 2 & $\begin{array}{c}\text { Peri-pancreatic } \\
\text { tissues, cyst }\end{array}$ & $(+)$ & $(-)$ & $(-)$ & $\begin{array}{c}\text { SMA, desmin, } \\
\text { caldesmon-focally } \\
(+)\end{array}$ & $(+)$ & Single cells & Focal & - \\
\hline 3 & $\begin{array}{l}\text { Small bowel, } \\
\text { perforation }\end{array}$ & $(+)$ & $(-)$ & & SMA $(-)$, desmin $(-)$ & $(+)$ & - & - & - \\
\hline 4 & $\begin{array}{l}\text { Mesentery, } \\
\text { diffuse }\end{array}$ & $(+)$ & $(-)$ & & $\operatorname{Desmin}(+)$ & $(+)$ & Single cells & Single cells & - \\
\hline 5 & Sole & $(+)$ & & & & & & & \\
\hline
\end{tabular}


Table II. Stainings advised in differential diagnosis of fibromatosis

\begin{tabular}{lll}
\multicolumn{1}{c}{ Positive stainings } & Negative stainings & \multicolumn{1}{c}{ Focal reaction $(+/-)$} \\
\hline - $\beta$-Catenin-mandatory for & - S100 & - Desmin \\
diagnosis (positive nuclear reaction & $\cdot$ CD34 & - Caldesmon \\
in majority of cells in DTF) & CD99 & - SMA \\
- Vimentin & - Bcl2 & - CD117 (single cells) \\
& & - ER, PGR (results of this study)
\end{tabular}
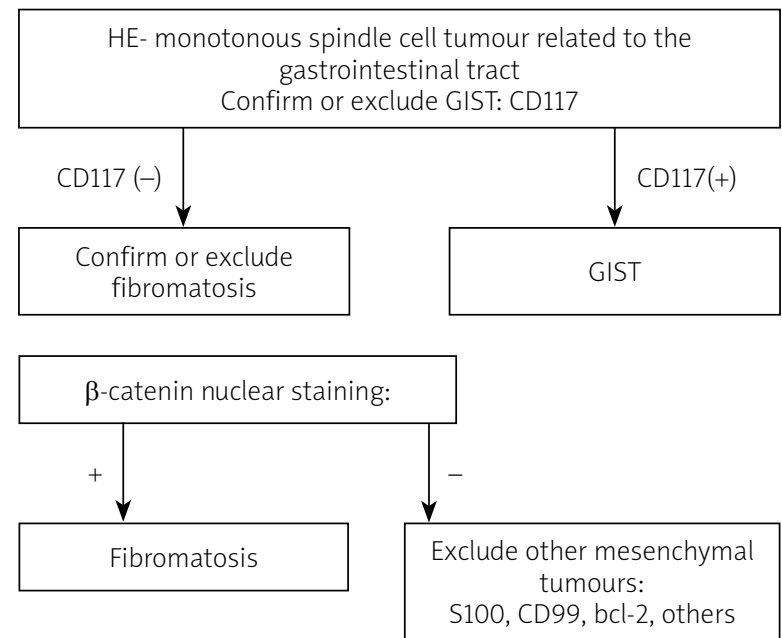

Figure 9. Short algorithm of immunostainings advised in differential diagnosis of fibromatosis, GIST, and other mesenchymal tumours

mesentery or bowel wall, as was seen in the described cases. Immunohistochemical stainings with CD117 and other antibodies are useful in differential diagnosis and should be performed whenever a suspicion of GIST occurs (Table II). One must also remember that negative CD117 and DOG1 in a solitary spindle cell tumour do not justify the diagnosis of wild type GIST, as was demonstrated in the first described case. In such instances $\beta$-catenin is an obligatory staining and is mandatory for confirming fibromatosis, although a minority of GIST tumours may show some nuclear positive staining. Nevertheless, in the case of deep fibromatosis, usually most of the nuclei are positive.

Overabundant fibrosis and Ormond's disease (idiopathic abdominal fibrosis) always have to be excluded while diagnosing fibromatosis. Immunohistochemical stainings such as vimentin, SMA, and caldesmon, which are known usually to be positive in deep fibromatosis, may be helpful (Table II).

Deep type fibromatosis (DTF) should present negative staining for S100, CD34, CD99, and bcl-2, which can help in distinguishing it from low-grade neurofibrosarcoma and solitary fibrous tumour.

Treatment of desmoid fibromatosis is multidisciplinary, and due to its unpredictable clinical course it should be individualised. Surgery is the basis of treatment with the goal of negative margins, which can sometimes be difficult to obtain, as was illustrated in two of our cases. However, the recurrence can happen even in patients with negative margins; therefore, NCCN guidelines recommend observation and watchful waiting rather than excision in patients with small tumours. If the patient is asymptomatic and surgery could cause major complications, watchful waiting is also advised [10].

Results of neoadjuvant radiotherapy in DTF are still not well established $[11,12]$. The NCCN recommends post-operative radiation for large DTF, but clinicians must be aware that radiation therapy may result in secondary malignancies. Additional systemic therapy includes hormonal therapy, non-steroidal anti-inflammatory drugs, tyrosine kinase inhibitors, and cytotoxic therapy as well as interferon, but there are still few data on the success of such treatment [5]. Nevertheless, the expression of hormonal receptors in described cases was only focal or negative.

It is also advised that patients with the diagnosis of intraabdominal desmoid tumour are evaluated for Gardner syndrome.

Further studies on the genetics of this disease may reveal its pathogenesis and thus help in finding better treatment options.

\section{Conclusions}

The analysis in this study showed the importance of differential diagnosis in young patients between GIST and abdominal fibromatosis as well as other mesenchymal tumours [9]. As the result of the study we propose an immunohistochemical algorithm that can be useful in differential diagnosis of spindle cell tumors of the abdomen that include fibromatosis, GIST, and mesenchymal tumours (Figure 9).

\section{Conflict of interest}

The authors declare no conflict of interest.

\section{References}

1. Diagnostic Histopathology of Tumors: 2 Volume Set. Fletcher. CDM (ed.). Churchill Livingstone 2013. 
2. Surgical Pathology of the GI Tract, Liver, Biliary Tract, and Pancreas. Odze RD, Goldblum JR, Crawford JM (eds). Saunders 2008.

3. Seow-Choen F. The management of desmoids in patients with familial adenomatous polyposis (FAP). Acta Chirur Iugoslav 2008; 55: 83-7.

4. Hughes DP, Kummar S, Lazar AJ. New, tolerable gamma-secretase inhibitor takes desmoid down a notch. Clin Cancer Res 2015; 21: 7-9.

5. Mignemi NA, Itani DM, Fasig JH, et al. Signal transduction pathway analysis in desmoid-type fibromatosis: transforming growth factor-beta, COX2 and sex steroid receptors. Cancer Sci 2012; 103: 2173-80.

6. van Broekhoven DL, Verhoef C, Grünhagen DJ, et al. Prognostic value of CTNNB1 gene mutation in primary sporadic aggressive fibromatosis. Ann Surg Oncol 2015; 22: 1464-70.

7. Montgomery E, Lee JH, Abraham SC, Wu TT. Superficial fibromatoses are genetically distinct from deep fibromatoses. Mod Pathol 2001; 14: 695-701.

8. Robanus-Maandag E, Bosch C, Amini-Nik S, et al. Familial adenomatous polyposis-associated desmoids display significantly more genetic changes than sporadic desmoids. PLoS One 2011; 6: e24354.

9. Liu X, Zong S, Cui Y, Yue Y. Misdiagnosis of aggressive fibromatosis of the abdominal wall: a case report and literature review. Medicine 2018; 97: e9925.

10. Wysocki WM, Wysocka J, Rutkowski P. Postępowanie w przypadku sporadycznej włókniakowatości typu desmoidu (stanowisko EORTC i SPAEN z 2014r.). Med Prakt Onkol 2015; 3: VII-IX.

11. Smith K, Desai J, Lazarakis S, Gyorki D. Systematic review of clinical outcomes following various treatment options for patients with extraabdominal desmoid tumors. Ann Surg Oncol 2018; 25: 1544-54.

12. Al-Jazrawe M, Au M, Alman B. Optimal therapy for desmoid tumors: current options and challenges for the future. Exp Rev Anticancer Ther 2015; 15: 1443-58.

Received: 28.04.2018

Accepted: 20.06 .2018 